\title{
Cambridge Ophthalmological Symposium 2018: introduction and reflections on the day
}

\author{
Marian Ludgate ${ }^{1}$
}

Received: 23 November 2018 / Accepted: 23 November 2018 / Published online: 19 December 2018

(c) The Royal College of Ophthalmologists 2018

\begin{abstract}
I was privileged to be one of the co-chairs, along with Professor Tim Sullivan (Brisbane, Australia), for the Cambridge Ophthalmological Society (COS) annual international symposium, which, this year, was dedicated to thyroid eye disease (TED). Together with the organisers, Miss Rachna Murthy and Professor Keith Martin from COS, we compiled an impressive programme covering all aspects of the condition from events happening in a single orbital cell to improved surgical approaches.
\end{abstract}

\section{Introduction}

In my introduction on the first day, which was dedicated to basic science, I provided a broad overview of the 'state-ofthe-art'. Thyroid eye disease (TED) is an autoimmune condition most often associated with hyperthyroid Graves' disease (GD). In GD, autoantibodies to the thyrotropin receptor (thyroid-stimulating hormone receptor, TSHR) mimic the biological action of thyroid-stimulating hormone, resulting in hyperthyroidism [1]. Previously considered to be thyroid specific, many studies have demonstrated that TSHR is expressed in orbital tissues [2]. Our own studies have reported that TSHR is expressed in orbital fat [3] and is upregulated during the lineage-specific differentiation of mesenchymal stem cells into adipocytes [4]. This process is known as adipogenesis and is one of the mechanisms, along with over-production of extra-cellular matrix (ECM), especially hyaluronan (HA), that leads to the expansion of the orbital contents [2]. The expansion occurs within an inflexible orbit and results in proptosis; indeed, even patients with less-severe exophthalmos may suffer sight-threatening optic neuropathy [5]. The extra-ocular muscles may become fibrosed and this can cause problems with eye motility; fibrosis may be considered a life-long condition [6].

Marian Ludgate

Ludgate@cardiff.ac.uk

1 Professor Emerita, Institute of Infection \& Immunity, School of Medicine, Cardiff, UK
The fact that some TED patients are euthyroid and/or apparently free of TSHR autoantibodies has led some investigators to seek additional thyroid/orbit-shared autoantigens. The IGF1R and its signalling pathways have been a subject of intense investigation, often with conflicting results. Smith and Janssen have demonstrated that autoantibodies to IGF1R in TED patients produce a range of effects, including enhanced OF proliferation, increased secretion of inflammatory cytokines and elevated production of glycosaminoglycans (GAGs) [7]. Others find little evidence for IGF1R autoantibodies being elevated in TED [8], while Neumann and co-workers suggest that cross-talk between TSHR and IGF1R, following the activation of TSHR, has a central role in TED pathogenesis [9]. This model could be compatible with the dramatic improvements in proptosis following treatment with an IGF1R antagonist, teprotumumab, reported by Smith et al. [10].

Much TED research in recent years has been focussed on the end stages of the disease, and relatively little progress has been made in understanding the mechanisms that break tolerance to TSHR. Several hypotheses propose that microorganisms might be implicated either via molecular mimicry or via bystander activation $[11,12]$. These hypotheses have been modified by the recognition that commensal organisms in the gut microbiota secrete metabolites, which influence the phenotype of $\mathrm{T}$ cells in the gut-associated lymphoid tissue. Approximately $50 \%$ of circulating $\mathrm{T}$ cells reside within the gut at any time, and studies in murine models of autoimmunity and human disease suggest that the gut microbiota may influence the balance between inflammatory Th17 and anti-inflammatory Treg [13, 14]. 
Thus, we are at an exciting point in TED research; there are many unanswered questions, plenty of controversies and the very real promise of novel medical therapies. I considered all of these aspects when compiling the programme for the basic science day of the symposium.

It seemed logical to 'take it from the top' and start in the orbit. Who better than Drs. Maryse Baillye and Lei Zhang have applied in vitro models to unravel the tissueremodelling processes at play in TED? Maryse is an expert on fibrosis, which remains one of the least-wellunderstood aspects of TED, and as noted above, can last forever [6]. She recently reported on the stem-cell properties of orbital fibroblasts [15] and also has a superb 3D in vitro model that will be invaluable in dissecting how pressure influences the disease [16], many TED patients having shown increased intra-ocular pressure. Lei has demonstrated that the processes of adipogenesis and ECM expansion via HA production are linked in the orbit but not in other fat depots. He has extended this finding to identify the possible targets for non-immunosuppressive TED therapy [17-20].

Teprotumumab is a human monoclonal antibody that binds with IGF1R and was originally developed to treat cancer. When applied to the treatment of TED it improves proptosis and thus must affect adipogenesis, proliferation, fibrosis and/or ECM production either directly or indirectly [10]. I was delighted that Prof. Terry Smith accepted our invitation to describe this seminal clinical trial and the science that underpins it. I was equally delighted that Prof. Susanne Neumann also agreed to cross the Atlantic to explain the work of her team on TSHR-IGF1R cross-talk [9]. Prof. Neumann's group has also developed smallmolecule TSHR antagonists [21, 22], which might provide an adjunct or combination therapy for TED.

The thyroid stimulating antibodies (TSABs) that cause GD are produced by plasma cells, which are short-lived and derived from B cells, and they all circulate in the blood, our next topic for discussion. Prof. Mario Salvi was one of the first to conduct trials using rituximab, a chimeric human/ mouse monoclonal antibody that targets CD20 on B cells resulting in their depletion and improves TED in some patients [23]. The benefits noted cannot all be explained by the reduction in TSAB titres, and this hints at the important additional roles of $\mathrm{B}$ cells including antigen presentation to $\mathrm{T}$ cells and secretion of pro-inflammatory cytokines [24].

It is a common experience that not all patients respond to a particular therapy in the same way, and what works well for one individual might have no effect on another. This reflects the heterogeneity of the processes operating in TED, with older patients more likely to have 'big muscles' and younger patients 'big fat' [2]. Hence it would be helpful to identify circulating biomarkers that aid assignment to the most appropriate treatment. Dr. Filippo Biscarini and co-workers have recently published a study that applied proteomic and genomic (miRNA) profiling of serum/plasma to compare TED of GD patients and matched healthy controls [25].

Most immunologists would argue that $\mathrm{T}$ cells are the main drivers of all immune responses, including autoimmune reactions. In this context, an intriguing human model of GD/TED is provided by patients with relapsing/ remitting multiple sclerosis (MS) in the immunereconstitution phase following treatment with alemtuzamab (ALTZ). ALTZ is a humanised monoclonal antibody to CD52, an antigen on the surface of all $\mathrm{T}$ and $\mathrm{B}$ cells that is consequently eliminated by the drug. It has been proved beneficial in MS, but in the 2-5 years following treatment, B cells are the first to reappear, often in higher numbers than pre-treatment, followed by $\mathrm{T}$ cells, which rarely attain pre-treatment levels. In the same time-frame, approximately 30-40\% develop GD, of which 5-10\% also have TED [26]. Dr. Joanne Jones has studied the mechanisms operating during immune reconstitution, which should be highly relevant to spontaneous GD and TED [27].

The second speaker on $\mathrm{T}$ cells was Prof. Li Bin, who works on fibrosis, which follows myofibroblast differentiation of CD90/Thy-1 + ve orbital fibroblasts, stimulated by TGF-beta. Recently, a role for Th17 cells has been implicated in fibrosis, in line with TH17 cells being found in autoimmune lesions, e.g. MS plaques. Li Bin's work [28] showed a significantly higher proportion of IL-17Aproducing $\mathrm{T}$ cells in TED patients and the recruitment of both CD $4+$ and CD8 + T cells in TED orbits. TED orbital tissues expressed more IL-17A receptor, IL-17A, and its related cytokines, with severe fibrotic change compared with normal controls. Unfortunately, visa problems precluded Prof. Bin from attending the symposium, and I had looked forward to discussing other aspects of his work in which a product isolated from a traditional Chinese medicine (Vialinin A) was able to inhibit Ror $\gamma \mathrm{T}$, a transcription factor that drives Th17 differentiation and thus reduces their numbers [29].

These last findings are of interest in light of current efforts to understand how the gut microbiota composition influences health and disease. Prof. Julian Marchesi has extensive experience in the metagenomic and metataxonomic methodology required to analyse microbiota, which he originally applied to soil ecology. More recently he has been central to investigations of the gut and other microbiomes (skin, vagina etc.) in a wide range of human conditions from cancer to cirrhosis and foetal pre-term membrane rupture. In this context, it is recognised that the gut microbiota is impacted by a variety of factors including diet, antibiotic use, the mode of delivery and whether you were breast- or bottle-fed as an infant. He has collaborated with me on a recently completed European Union (EU)-funded project the goal of which was to assess 
whether the gut microbiome is implicated in GD and/or TED (http://www.indigo-iapp.eu/).

The last three speakers use in vivo models of the disease and began with Dr. David Williams, whose work in zebrafish and other amphibians has yielded further understanding of the development of the eye.

In other autoimmune conditions, in vivo models have provided an insight into the processes leading to loss of tolerance. My own experience with a TSHR-induced model (genetic immunisation) of GD/TED was frustrating; while we were able to transfer the disease to naive female BALB/c mice using TSHR-primed $\mathrm{T}$ cells in one location [30], we were not able to reproduce the model in another laboratory [31]; neither animal unit was specific pathogen free. Prof. Anja Eckstein and her co-workers have been able to reproduce a model [32] originally established by Sajad Moshkelgosha in Paul Banga's lab [33]. The model uses genetic immunisation and in vivo electroporation of the TSHR A subunit, and Prof. Eckstein has access to imaging methods able to evaluate induced TED over time and not just at the end point when the animal is sacrificed.

The in vivo model was also a part of the indigo project, and Ms. Giulia Masetti has analysed the gut microbiota composition of the mice with TSHR-induced disease in the two centres (London and Essen). She identified significant differences between the two centres, which might explain the heterogeneity of the response observed. She also found disease-associated taxonomies when comparing TSHRimmunised and control empty-vector-immunised mice in Essen [34]. Furthermore, there were many similarities between the gut microbiota composition in the induced murine model and that in patients with GD/TED (manuscripts in preparation).

\section{Reflections on the day}

So, what did we learn after the long but invigorating day? Many questions remain unanswered

(i) Fibrosis remains one of the biggest challenges faced by patients with TED and the clinicians who manage them. It causes disturbance to eye motility, which has a major negative impact on the quality of life, but we are not aware of any ongoing or pending trials to address this process. (ii) We need to recognise the limitations of our in vitro models, for e.g. many use M22, a human monoclonal TSAB, to assess the effects of TSHR activation on orbital fibroblastsbut the patient from whom it was derived does not have TED. (iii) The 'omics study is interesting but needs to be repeated on a much larger number of cases and controls; identified biomarkers then need to be validated using specific assays. (iv) Have we identified the autoantigen(s) driving TED? To be considered a relevant autoantigen, it should be (a) expressed in the orbit; (b) disease activity should correlate with antibody- or T cell-mediated immune response to the autoantigen; (c) TED-like disease should be induced using the autoantigen; and (d) antagonism of the autoantigen should improve the disease. Neither TSHR nor IGF1R ticks all four boxes; IGF1R is still awaiting (b) and (c), whilst TSHR does not yet fulfil (d); although treatment with a human monoclonal thyroid-blocking antibodyimproved GD and TED in an unusual patient with these disorders in addition to thyroid cancer (ETA abstract). These results are also compatible with my view that we do not need any additional autoantigens-but may be we have yet to identify TED-specific TSHR autoantibodies [35].

We are not as clever as we think

(i) Despite all our best efforts the incidence of people with TED who smoke remains high, as illustrated by the patient cohort studied in the search for circulating biomarkers. (ii) Preliminary results from the indigo human microbiome project demonstrated significant differences in gut microbiota composition among GD, TED and healthy controls. However, it is difficult to distinguish the contributions of endocrine (hyperthyroidism) and immune (TSAB) factors. While an additional group of patients with non-autoimmune hyperthyroidism might have been informative, it is hoped that the comparison of samples from the same patients at different time points will help to resolve this point. Would other microbiomes be more relevant? Indigo collected nasal swabs as a surrogate for the sinus microbiome, which are available for analysis-we are happy to collaborate.

\section{Promising therapies need validation}

(i) In many ways the results of the teprotumumab drug trial in TED were the highlight of the meeting. However, it needs to be repeated in a much larger series of patients to confirm the outcomes and also needs to be compared with the standard steroid therapy currently used to manage TED, before being extended to all suitable patientsespecially as it is very expensive. (ii) Immune-cell depletion studies are finding more application in selected patients. The usefulness of the ALTZ-induced human model of GD/TED is already apparent with the recent report that TSHR autoantibodies, mainly lacking biological function, can be detected in MS patients before the onset of induced GD and sometimes even before treatment [36]. Such autoantibodies may also be present long before clinical disease in spontaneous GD and TED. (iii) Dr. Paul Meyer has investigated the possible role of Staphylococcus aureus infection and its treatment with antibiotics in TED. His colleague, one of the symposium organisers, Dr. Rachna Murthy is analysing the data that should prove the most informative. 


\section{Novel treatment targets merit clinical trials}

(i) In vitro models employing orbital fibroblasts illustrated that the transcription factor FOXO1 is a convergence point for the signalling cascades stimulating adipogenesis and HA generation. When FOXO1 is located in the nucleus it inhibits these cascades; trifluoperazine inhibits the nuclear exclusion of FOXO1 and, at least in vitro, it is an effective inhibitor of adipogenesis and HA accumulation. The drug was developed as a cancer therapy and so has undergone phase 1 trials; it should be added to the list to be trialled in TED. (ii) TSHR antagonists hold considerable promise for the treatment of TED. In addition to the monoclonal thyroid- blocking antibody described above, small-molecule antagonists have been shown to be effective in vitro and are currently being tested in the TSHR-induced mouse model-we await the results with anticipation.

We did not have time to cover all the aspects of TED pathophysiology

(i) Smoking is the single biggest known environmental risk factor for TED incidence and severity [37]. In vitro studies from Tom Cawood illustrated that smoke extract enhances adipogenesis/GAG accumulation in orbital fibroblasts [38]. We applied MALDI-TOF mass spectrometry to analyse tears and found similar compositions in those from TED patients and healthy smokers, with excess zinc alpha-2 glycoprotein [39], also known as fat-mobilising factor, which has been shown to be upregulated in smokers [40]. Smoking affects the gut microbiota composition [41], but in this and all studies on the impact of smoking it remains unclear whether nicotine or smoke extracts are responsible. (ii) Selenium is a component of selenocysteine-containing proteins, which are mainly enzymes. This includes glutathione peroxidase, which helps protect against the negative effects of reactive oxygen species. Selenium supplementation improved the outcome of mild TED [42]. (iii) Platelet-derived growth factor, especially the plateletderived growth factor-BB isoform, stimulates proliferation and HA production, in both healthy and TED orbital fibroblasts [43]. (iv) Thyrostimulin is also known as corticotroph-derived glycoprotein hormone. It is a heterodimer of the novel alpha- 2 and beta- 5 subunits, and evidence to date suggests that it acts in a paracrine fashion, since it has not been detected in the circulation. Of interest to TED, mice overexpressing beta-5 have elevated T4, weight loss and proptosis [44].

In my career of $30+$ years I have attended many conferences dedicated to increasing understanding and improving the management of TED. Considerable strides have been made in enhancing patient outcomes, and I am optimistic that ongoing studies will lead to further advances. Such progress follows meetings such as that at St John's College, Cambridge, and I greatly appreciate COS and its benefactors for choosing TED as its topic.
Acknowledgements I am sincerely grateful to the Cambridge Ophthalmological Society and the organisers of the symposium for the privilege of chairing this meeting; special thanks to Louise.

\section{Compliance with ethical standards}

Conflict of interest The authors declare that they have no conflict of interest.

Publisher's note: Springer Nature remains neutral with regard to jurisdictional claims in published maps and institutional affiliations.

\section{References}

1. Bahn RS. Graves' ophthalmopathy. N Engl J Med. 2010;362: 726-38. 10.1056/NEJMra0905750 (2010).

2. Draman MS, Ludgate M. Thyroid eye disease-an update. Expert Rev Ophthalmol. 2016. https://doi.org/10.1080/17469899.2016. 1202113

3. Crisp M, Lane C, Halliwell M, Wynford-Thomas D, Ludgate M. Thyrotropin receptor transcripts in human adipose tissue. J Clin Endocrinol Metab. 1997;82:2003-5.

4. Crisp MS, Starkey KJ, Lane C, Ham J, Ludgate M. Adipogenesis in thyroid eye disease. Invest Ophthalmol Vis Sci. 2000;41: 3249-55.

5. Wiersinga WM, Kahaly GJ. Graves' orbitopathy: a multidisciplinary approach. 3rd edn. Karger; 2017.

6. Perros P, Hegedüs L, Bartalena L, Marcocci C, Kahaly GJ, Baldeschi L, et al. Graves' orbitopathy as a rare disease in Europe: a European Group on Graves' Orbitopathy (EUGOGO) position statement. Orphanet J Rare Dis. 2017;12:72.

7. Smith TJ, Janssen J. Insulin-like growth factor-I receptor and thyroid-associated ophthalmopathy. Endocr Rev. 2018. https://doi. org/10.1210/er.2018-00066

8. Minich WB, Dehina N, Welsink T, Schwiebert C, Morgenthaler NG, Köhrle J, et al. Autoantibodies to the IGF1 receptor in Graves' orbitopathy. J Clin Endocrinol Metab. 2013;98:752-60. https://doi.org/10.1210/jc.2012-1771

9. Krieger CC, Robert FPlace, Bevilacqua Carmine, MarcusSamuels Bernice, Abel BrentS, Monica C, et al. Bidirectional TSH and IGF-1 receptor cross talk mediates stimulation of hyaluronan secretion by Graves' disease immunoglobins. J Clin Endocrinol Metab. 2015;100:1071-7.

10. Smith TJ, Kahaly GJ, Ezra DG, Fleming JC, et al. Teprotumumab for thyroid-associated ophthalmopathy. N Engl J Med. 2007; 376:1748-61. https://doi.org/10.1056/NEJMoa1614949

11. Hogquist KA, Baldwin TA, Jameson SC. Central tolerance: learning self-control in the thymus. Nat Rev Immunol. 2005;5: $772-82$.

12. Mueller DL. Mechanisms maintaining peripheral tolerance. Nat Immunol. 2010;11:21-7.

13. Köhling H, Sue FPlummer, Marchesi JulianR, Davidge KellyS, Ludgate Marian. The microbiota and autoimmunity: their role in thyroid autoimmune diseases. Clin Immunol. 2017;183:63-74. https://doi.org/10.1016/j.clim.2017.07.001. I. I.

14. Covelli D, Ludgate M. The thyroid, the eyes and the gut: a possible connection. J Endocrinol Invest. 2017. https://doi.org/10. 1007/s40618-016-0594-6

15. Kozdon K, Fitchett C, Rose GE, Ezra DG, Bailly M. Mesenchymal stem cell-like properties of orbital fibroblasts in Graves' orbitopathy. Invest Ophthalmol Vis Sci. 2015; 56:5743-50. https://doi.org/10.1167/iovs.15-16580. PMID: 26325413 
16. Li H, Fitchett C, Kozdon K, Jayaram H, Rose GE, Bailly M, et al. Independent adipogenic and contractile properties of fibroblasts in Graves' orbitopathy: an in vitro model for the evaluation of treatments. PLoS ONE. 2014;9:e95586 https://doi.org/10.1371/ journal.pone.0095586

17. Zhang L, Ji QH, Ruge F, Lane C, Morris D, Tee AR, et al. Reversal of pathological features of Graves' Orbitopathy by activation of Forkhead transcription factors, FOXOs. J Clin Endocrinol Metab. 2016;101:114-22. https://doi.org/10.1210/jc.2015-2932

18. Zhang L, Grennan-Jones F, Draman MS, Lane C, Morris D, Dayan CM, et al. Possible targets for non-immunosuppressive therapy of Graves' orbitopathy. J Clin Endocrinol Metab. 2014;99:E1183-90.

19. Zhang L, Bowen T, Grennan-Jones F, Paddon C, Giles P, Webber $\mathrm{J}$, et al. Thyrotropin receptor activation increases hyaluronan production in preadipocyte- fibroblasts; contributory role in hyaluronan accumulation in thyroid dysfunction. J Biol Chem. 2009;284:26447-55.

20. Zhang L, Grennan-Jones F, Lane C, Rees DA, Dayan CM, Ludgate M. Adipose tissue depot specific differences in the regulation of hyaluronan production of relevance to Graves' orbitopathy. J Clin Endocrinol Metab. 2012;97:653-62.

21. Neumann S, Eliseeva E, McCoy JG, Napolitano G, Giuliani C, Monaco F, et al. A new small-molecule antagonist inhibits Graves' disease antibody activation of the TSH receptor. J Clin Endocrinol Metab. 2011;96:2152-929.

22. Turcu AF, Kumar S, Neumann S, Coenen M, Iyer S, Chiriboga P, et al. A small molecule antagonist inhibits thyrotropin receptor antibody-induced orbital fibroblast functions involved in the pathogenesis of Graves ophthalmopathy. J Clin Endocrinol Metab. 2013;98:2153-9.

23. Salvi M, Vannucchi G, Currò N, Campi I, Covelli D, Dazzi D, et al. Efficacy of B-cell targeted therapy with rituximab in patients with active moderate to severe Graves' orbitopathy: a randomized controlled study. J Clin Endocrinol Metab. 2015;100:422-31.

24. Mauri Claudia, Bosma Anneleen. Immune regulatory function of B cells. Annu Rev Immunol. 2012;30:221-41. https://doi.org/10. 1146/annurev-immunol-020711-074934

25. Zhang L, Masetti G, Colucci G, Salvi M, Covelli D, Eckstein A, et al. Combining micro-RNA and protein sequencing to detect robust biomarkers for Graves' disease and orbitopathy. Sci Rep. 2018. https://doi.org/10.1038/s41598-018-26700-1 https://rdcu. be/QmeM

26. Coles AJ, Wing M, Smith S, Coraddu F, Greer S, Taylor C, et al. Pulsed monoclonal antibody treatment and autoimmune thyroid disease in multiple sclerosis. Lancet. 1999;354:1691-5.

27. Jones JL, Thompson SA, Loh P, Davies JL, Tuohy OC, Curry AJ, et al. Human autoimmunity after lymphocyte depletion is caused by homeostatic T-cell proliferation. Proc Natl Acad Sci USA. 2013;110:20200-5

28. Fang $\mathrm{S}$, Huang $\mathrm{Y}$, Zhong $\mathrm{S}, \mathrm{Li} \mathrm{Y}$, Zhang $\mathrm{Y}, \mathrm{Li} \mathrm{Y}$, et al. Regulation of orbital fibrosis and adipogenesis by pathogenic th 17 cells in Graves orbitopathy. J Clin Endocrinol Metab. 2017;102:4273-83.

29. Fang S, Huang Y, Wang S, Zhang Y, Luo X, Liu L, et al. IL-17A exacerbates fibrosis by promoting the proinflammatory and profibrotic function of orbital fibroblasts in TAO. J Clin Endocrinol Metab. 2016;101:2955-65.

30. Many M-C, Costagliola S, Detrait M, Denef J-F, Vassart G, Ludgate M. Development of an animal model of autoimmune thyroid eye disease. J Immunol. 1999;162:4966-74.
31. Baker G, Mazziotti G, von Ruhland C, Ludgate M. Re-evaluating thyrotropin receptor induced mouse models of Graves' disease \& ophthalmopathy. Endocrinology. 2005;146:835-44.

32. Moshkelgosha S, Po-Wah S, Deasy N, Diaz-Cano S, Banga JP, et al. Cutting edge: retrobulbar inflammation, adipogenesis, and acute orbital congestion in a preclinical female mouse model of Graves' orbitopathy induced by thyrotropin receptor plasmidin vivo electroporation. Endocrinology. 2013;154:3008-15.

33. Berchner-Pfannschmidt U, Moshkelgosha S, Diaz-Cano S, Edelmann B, Görtz G-E, Horstmann M, et al. Comparative assessment of female mouse model of Graves' orbitopathy under different environments, accompanied by pro-inflammatory cytokine and $\mathrm{T}$ cell responses to thyrotropin hormone receptor antigen. Endocrinology. 2016: p. en20151829.

34. Masetti G, Moshkelgosha S, Köhling HL, Covelli D, Banga JP, Berchner-Pfannschmidt U, et al. Gut microbiota in experimental murine model of Graves' orbitopathy established in different environments may modulate clinical presentation of disease. Microbiome. 2018. https://doi.org/10.1186/s40168-018-0478-4 https://microbiomejournal.biomedcentral.com/track/pdf/10.1186/ s40168-018-0478-4

35. Metcalfe R, Jordan N, Watson P, Gullu S, Wiltshire M, Crisp M, et al. Demonstration of $\operatorname{IgG}, \operatorname{IgA}$ and $\operatorname{IgE}$ autoantibodies to the human thyrotropin receptor using flow cytometry. J Clin Endocrinol Metab. 2002;87:1754-61.

36. Muller I, Willis M, Healy S, Nasser T, Loveless S, Butterworth S, et al. Longitudinal characterization of autoantibodies to the thyrotropin receptor (TRAb) during alemtuzumab therapy; evidence that TRAb may precede thyroid dysfunction by many years. Thyroid. 2018 Oct 23. https://doi.org/10.1089/thy.2018.0232. [Epub ahead of print]

37. Wiersinga WM. Smoking and thyroid. Clin Endocrinol (Oxf). 2013;79:145-51. https://doi.org/10.1111/cen.12222

38. Cawood TJ, Moriarty P, O'Farrelly C, O'Shea D. Smoking and thyroid-associated ophthalmopathy: A novel explanation of the biological link. J Clin Endocrinol Metab. 2007;92:59-64. https:// doi.org/10.1210/jc.2006-1824

39. Baker GRC, Morton M, Rajapaska RS, Bullock M, Gullu S, Mazzi B, et al. Altered tear composition in smokers and patients with Graves' ophthalmopathy. Arch Ophthalmol. 2006;124: 1451-6.

40. Vanni H, Kazeros A, Wang R, Harvey BG, Ferris B, De BP, et al. Cigarette smoking induces overexpression of a fat-depleting gene AZGP1 in the human. Chest. 2009;135:1197-208. https://doi.org/ 10.1378/chest.08-1024. PMC2679098. PMID 19188554

41. Savin Z, Kivity S, Yonath H, Yehuda S. Smoking and the intestinal microbiome. Arch Microbiol. 2018;200(Issue 5): 677-84.

42. Claudio Marcocci, George JKahaly, Gerasimos EKrassas, Luigi Bartalena, Mark Prummel, et al. Selenium and the course of mild Graves' orbitopathy. N Engl J Med. 2011;364:1920-31. https:// doi.org/10.1056/NEJMoa1012985

43. Virakul S, van Steensel L, Dalm VA, Paridaens D, van Hagen PM, Dik WA. Orbit-infiltrating mast cells, monocytes, and macrophages produce PDGF isoforms that orchestrate orbital fibroblast activation in Graves' ophthalmopathy. J Clin Endocrinol Metab. 2012;97:E400-408. https://doi.org/10.1210/jc.2011-2697

44. Okada SL, Jeff L Ellsworth, Diane M Durnam, Harald S Haugen, James L Holloway, Merideth L Kelley et al. A glycoprotein hormone expressed in corticotrophs exhibits unique binding properties on thyroid-stimulating hormone receptor. Mol Endocrinol. 2006; 20:414-25. 